\title{
TRADUCCIONES EN VERSO DEL SIGLO XV
}

\author{
Marta Marfany \\ Universitat Pompeu Fabra
}

Entre 1304 y 1307, Dante Alighieri escribió Il Convivio, un ambicioso proyecto enciclopédico truncado, pues sólo completó cuatro de los catorce tratados previstos. En cada tratado Dante presenta un poema suyo seguido de un comentario en prosa, en cuyos capítulos se desarrollan temas filosóficos, morales y científicos. La obra, que tenía el propósito de divulgar el conocimiento más allá del entorno académico, se definía como un banquete de sabiduría: el autor servía un manjar exquisito y selecto que sus comensales sólo podían saborear con el acompañamiento del pan de la prosa (Il Convivio, I, 1). Puesto que su objetivo era llegar a lectores instruidos con afán de conocimiento pero sin estudios superiores, Dante redactó la obra en toscano y no en latín como era habitual en los tratados filosóficos. En su justificación del uso de la lengua vulgar, Dante arguyó, entre otras consideraciones, que el latín habría difundido la obra más allá del público románico ('lo latino l'averebbe esposte a gente d'altra lingua, sí come a Tedeschi e Inghilesi e altri’; Il Convivio: I, 7, §13), lo cual habría supuesto un gran inconveniente para su poesía, porque 'sarebbe essere esposta la loro sentenza colà dov'elle non la potessero colla loro bellezza portare' (Il Convivio: I, 7, §13). La mención de alemanes e ingleses demuestra que la cuestión para Dante no era la difusión de su poesía entre un público no itálico, sino específicamente entre un público no románico. No en balde ya había señalado, en un pasaje anterior del mismo capítulo, la distancia entre las lenguas romances y las anglo-germánicas: '[...] chè uno abituato di latino non distingue, s'elli è d'Italia, lo volgare [inghilese] da lo tedesco; nè lo tedesco, lo volgare italico dal provenzale’ (Il Convivio: I, 7, §8). Los alemanes, ingleses y otros 
pueblos, pues, habrían captado el sentido del texto poético gracias a la prosa latina, pero el desconocimiento del toscano les habría impedido percibir la belleza del poema, es decir, su forma, su ritmo, su musicalidad. Para no dar lugar a dudas, Dante postuló la intraducibilidad de la esencia de la poesía:

E però sappia ciascuno che nulla cosa per legame musaico armonizzata si può della sua loquela in altra transmutare sanza rompere tutta sua dolcezza ed armonia. E questa è la cagione per che Omero non si mutò di greco in latino, come l'altre scritture che avemo da loro. E questa è la cagione per che i versi del Salterio sono sanza dolcezza di musica e d'armonia: ché essi furono transmutati d'ebreo in greco e di greco in latino, e nella prima transmutazione tutta quella dolcezza venne meno. (Il Convivio: I, 7, §14-15)

Las palabras de Dante reflejan la concepción sobre la traducción poética imperante en la Edad Media. La posibilidad de trasladar esa 'dolcezza ed armonia' ni se contemplaba para los textos poéticos clásicos, que se traducían a las lenguas vulgares en prosa: el prestigio cultural del latín y la subsiguiente necesidad de glosar el saber elevado que el texto conllevaba excluían cualquier planteamiento traductológico que no se basara únicamente en el traslado y la explanación del sentido. ${ }^{1}$ A su vez, la traducción poética entre lenguas romances, próximas lingüística y culturalmente, era inexistente -adaptaciones y recreaciones aparte-, porque, para expresarlo en los términos de Dante, ya se podía apreciar la bellezza y entenderse la sentenza sin

\footnotetext{
${ }^{1}$ La traducción en prosa de los textos poéticos clásicos era un fenómeno general en todas las lenguas vulgares. Baste citar como ejemplo dos traducciones al catalán: la anónima de las Tragedias de Séneca, de finales del siglo XIV o principios del XV, y la que hizo Guillem Nicolau en 1390 de las Heroidas de Ovidio, versiones en prosa que integran, en grados distintos, pasajes de glosas o de comentarios escolásticos a las obras originales, una práctica medieval muy común. La traducción del latín al vulgar no era un traslado de igual a igual, puesto que el romance se percibía a un nivel lingüístico y cultural inferior (véase: Badia 1994).
} 
necesidad de traducción. ${ }^{2}$ Sin embargo, a partir del siglo XV se desarrolló la traducción de poesía en verso entre lenguas vulgares, un fenómeno que se generalizará a principios del siglo XVI. Ciertamente, a pesar de las fronteras altamente difusas entre obra original, adaptación y traducción a lo largo de la Edad Media, el fenómeno tenía precedentes ilustres, pues la canción Madonna dir vo voglio del siciliano Giacomo da Lentini (c.1210-c.1250) traduce al menos las dos estrofas conservadas de la canso del trovador Folquet de Marselha A vos, midonç, voill retrair'en cantan, $^{3}$ y en el siglo XIV la lengua inglesa ya contaba con las traducciones de Geoffrey Chaucer (1343-1400) de algunos poemas franceses. ${ }^{4}$

La primera gran traducción poética entre lenguas romances - con entidad propia, con finalidad estética y en verso- es precisamente, a pesar de la categórica declaración de Il Convivio, una traducción de la Commedia de Dante: el poeta Andreu Febrer (c.1375-1440/1444), al servicio del rey de Aragón Alfonso el Magnánimo, la tradujo al catalán en 1429. Apenas un año antes, Enrique de Villena (1384-1434) había traducido el Inferno al castellano, pero lo había hecho en prosa, como si de un clásico latino se

\footnotetext{
${ }^{2}$ Dante expuso su teoría sobre el latín y las lenguas románicas en De vulgari eloquentia (1302-1305), donde describe una tradición poética común a toda la Romania: 'Est igitur super quod gradimur ydioma tractando tripharium, ut superius dictum est: nam alii $o c$, alii sì, alii vero dicunt oil. Et quod unum fuerit a principio confusionis [...] apparet, quia convenimus in vocabulis multis, velut eloquentes doctores ostendunt [...] Trilingues ergo doctores in multis conveniunt, et maxime in hoc vocabulo quod est "amor".' (De vulgari eloquentia: I, 9, §2-3); el argumento se acompaña con citaciones de versos de Giraut de Bornelh, Thibaut de Champagne i Guido Guinizzelli, que ilustran las semejanzas entre la lírica de $o c$, la de oil i la de sì.

${ }^{3}$ Giacomo da Lentini no reproduce la métrica original del poema: las dos coblas unissonans (ABBAACDDCCD) de once versos decasílabos de Folquet de Marselha son trasladadas a estrofas de dieciséis versos, con secciones de tres heptasílabos y un endecasílabo, y se pasa de las cuatro rimas originales por estrofa a nueve.

${ }^{4}$ En la Complaint of Venus, Chaucer tradujo, reproduciendo la métrica original, tres baladas del poeta Oton de Grandson (c.1345-1397), al final de las cuales insirió un envoi justificativo: 'And eek to me hit is a greet penaunce, / Sith rym in English hath swich scarsitee, / To folowe word by word the curiositee / Of Graunson, flour of hem that make in France' (Piaget 1941: 174). Cabe descartar la función principalmente utilitaria de las traducciones de Chaucer: la nobleza inglesa de la Baja Edad Media conocía perfectamente la lengua francesa y su poesía, pues el francés había sido hasta el siglo XIII la lengua de la corte, gozó de un enorme prestigio cultural durante los dos siglos siguientes y saber francés fue siempre signo de distinción cortesana (el francés, junto con el latín, aún era en el siglo XV la lengua de la documentación oficial). También se atribuye con bastante seguridad a Chaucer la primera parte de The Romaunt of the Rose, una versión parcial del Roman de la Rose de Guillaume de Lorris y Jean de Meun (1225-1270).
} 
tratara. ${ }^{5}$ En efecto, la reverencia que sentían por Dante los autores del siglo $\mathrm{XV}$, que lo equiparaban a Homero y Virgilio, justifica en parte tanto la prosificación de Villena como la fidelidad métrica de Febrer, que vertió al catalán las terzine dantescas. Trasladar el endecasílabo italiano al equivalente decasílabo catalán y mantener la estructura encadenada de la terza rima era un desafío, una constricción métrica que Febrer se impuso en la ya de por si ardua tarea de traducir una obra tan compleja como la Commedia. $^{6}$ Respeto y veneración por el original explican la reproducción de la métrica, pero una empresa tan audaz sólo podía concebirla un poeta sobresaliente en la versificación como Febrer, que había renovado la poesía catalana experimentando con el tradicional trobar ric y adaptando las modernas formas fijas francesas. ${ }^{7}$ La Commedia catalana es fiel y exacta en muchos pasajes y, si bien algunas soluciones hoy nos parecen censurables, Febrer consiguió que no se disiparan ni el sentido ni la música de los tercetos italianos. ${ }^{8}$ La solución métrica de la Commedia catalana, no obstante, es un caso excepcional que diverge de las otras traducciones cuatrocentistas, confeccionadas con patrones distintos.

Es el caso de otro texto elaborado en la Corona de Aragón, la traducción catalana de La Belle Dame sans merci de Alain Chartier, hecha por Francesc Oliver a

\footnotetext{
5 Para un análisis de la traducción, con conclusiones importantes sobre la función del texto de Villena, véase Pascual 1974. Por otro lado, cabe destacar que en 1428 Enrique de Villena había traducido también en prosa la Eneida de Virgilio, la primera traducción de esta obra a una lengua romance. De linaje catalán por parte de padre, Enrique de Villena fue educado en tierras catalanas, dónde pasó luego, una vez establecido en Castilla, largas temporadas, y mantuvo siempre una estrecha relación con la Corona de Aragón. Para la formación cultural catalana de Enrique de Villena, en relación también con su faceta de traductor, véase: Gascón 1992. En 1417, estando en Valencia, Enrique de Villena concluyó la redacción en catalán de Els dotze treballs d'Hèrcules, que él mismo tradujo después al castellano.

${ }^{6}$ El poeta castellano Iñigo López de Mendoza, marqués de Santillana, que entre 1412 y 1418 formó parte de la corte catalanoaragonesa de Alfonso el Magnánimo, puso de relieve en su Prohemio e carta (1448/49) el reto métrico de la traducción catalana de la Commedia: 'Mosén Febrer fizo obras nobles, e algunos afirman aya traído el Dante de lengua florentina en catalán, no menguando punto en la orden de metrificar e consonar' (1997: 22). El marqués de Santillana, pues, no conoció de primera mano la traducción catalana de la Commedia, pero sí la castellana, ya que Enrique de Villena se la había dedicado. 7 Además de hábil versificador, Andreu Febrer era un buen lector y un gran poeta, pues sus poesías muestran la recreación sutil de Arnaut Daniel, Guillaume de Machaut y Dante. Para un análisis detallado de estas cuestiones y una visión general de la figura literaria de Andreu Febrer, véase: Cabré 2007.

${ }^{8}$ Para la traducción catalana de la Commedia, véase: Gallina 1974-1988 y Parera 2010.
} 
mediados del siglo XV. Las obras de Alain Chartier (1385/95-1430), secretario, embajador y consejero personal del rey Carlos VII de Francia, escritor de prestigio en latín y en francés, tuvieron una gran difusión en toda Europa. Su mayor éxito fue el poema cortesano La Belle Dame sans merci (1424), de ochocientos versos, que es esencialmente el diálogo entre un caballero enamorado y una dama despiadada que lo rechaza. Era el tipo de argumento à la page en las cortes de la época, y entusiasmó a la nobleza y a los poetas franceses, generando un intenso debate literario que desencadenó imitaciones, continuaciones directas y secuelas. Se convirtió rápidamente en un bestseller europeo y fue el texto francés más influyente del siglo XV, 'the most important single poem of its century in terms of literary history, and one of the finest in aesthetic terms as well' (Calin 1994: 250). No en balde fue objeto de tres traducciones en verso: además de la versión catalana, Sir Richard Roos tradujo el poema al inglés (c.14301442) y Carlo del Nero al italiano (1471). ${ }^{9}$

La elección métrica de los tres traductores de Chartier está condicionada en gran parte por la naturaleza cortesana del texto: un poema de amor, que además era archiconocido y se leía en la lengua original, sólo tenía sentido traducirlo con voluntad estética, adaptándolo a la lengua poética propia del traductor, que llevaba implícita formas métricas distintas en cada tradición. Así pues, el huitain libre que utiliza Chartier, una estrofa de ocho versos octosílabos con tres rimas, se traslada en la traducción catalana a octavas de versos decasílabos, la forma predominante en la poesía catalana del siglo XV, prestigiada por Ausiàs March, el principal poeta del cuatrocientos catalán. También acomodó el texto a su propia tradición el traductor italiano, que

\footnotetext{
${ }^{9}$ Para el original, véase: Laidlaw 1974; para la traducción catalana: Riquer 1983 y Marfany 2010; para la traducción italiana: Sansone 1997; y, para la traducción inglesa: Symons 2004 (los versos de La Belle Dame sans merci y de sus traducciones citados en este artículo proceden de estas ediciones). Por otro lado, un manuscrito de La Belle Dame sans merci conserva una traducción al latín interlineada al texto francés: el códice fue un encargo de Federico III de Sajonia (1463-1525) para promover el estudio del francés en la corte (Laidlaw 1974: 72); así pues, la traducción latina tenía una función puramente utilitaria.
} 
convirtió el huitain de La Belle Dame sans merci en terza rima, los tercetos encadenados endecasílabos de la Commedia de Dante y de otros textos célebres como los Triomphi de Petrarca. ${ }^{10}$ A su vez, el traductor inglés mantuvo, como el traductor catalán, la octava, pero adecuó el octosílabo francés al pentámetro yámbico, instaurado por el gran Geoffrey Chaucer.

En cierta medida, la elección de la estrofa determina el grado de literalidad de la traducción. Las traducciones inglesa y catalana siguen el poema francés de manera más o menos textual, puesto que el mantenimiento de la octava favorece el traslado verso a verso. En cambio, el traductor italiano se ve forzado a ampliar la octava francesa en tres terzine -nueve versos- o hasta incluso en cuatro -doce versos- y a reconstruir la estrofa recolocando o adaptando las ideas expresadas en la octava original. En ocasiones las licencias del traductor italiano contrastan con una mayor literalidad de los traductores inglés y catalán, como se puede apreciar en las traducciones de la octava XCVIII, donde el narrador cuenta el trágico final del enamorado:

\footnotetext{
Depuis je ne sceu qu'il devint

Ne quel part il se transporta;

Mais a sa dame n'en souvint

Qui aux dances se deporta.

Et depuis on me rapporta

Qu'il avoit ses cheveux desroux,

Et que tant se desconforta

Qu'il estoit mort de courroux.
}

(vv. 777-784)

\footnotetext{
${ }^{10}$ Carlo del Nero también tradujo Le Débat de reveille matin de Chartier, y lo hizo del mismo modo que para La Belle Dame sans merci, convirtiendo el huitain libre en terza rima.
} 
Fro thens he went, but whider wist I nought, Despuys no scé què·s féu, ne quina via

Nor to what part he drow, in sothfastnesse ne·n quina part aquell se trespostà;

But he no more was in his ladies thought, mas, certes, res no·n membrà a s'aymia,

For to the daunce anon she gan her dresse. ans en dançar e plaer se deportà.

And afterward, oon tolde me thus expresse, Mas enaprés nove $m$ fou reportada

He rente his heer, for anguissh and for payne,que los cabells s'ach romputs malament,

And in him-self took so gret hevinesse

That he was deed, within a day of twayne.

(vv. 806-813) e que per dol e vida maltractada

ell era mort de tristor e turment.

(vv. 777-784)
Non so quel ch'e si fu del giovanetto, né in che luogo si fu trasportato, privo di gioia e piacere e diletto. Alla sua dama poco n'è curato: torna a danzare e prender suo disporto.

E secondo mi fu poi reportato

el giovanne pigliò tanto isconforto per questa donna disperato e tristo, di cruccio e d'ira fu trovato morto.

(vv. 873-881)

A simple vista, en la traducción italiana se observa un verso añadido que no corresponde a ninguno del original, 'privo de gioia e piacere e diletto' (v. 875), amplificación del anterior. Además, el verso francés 'qu'il avoit ses cheveux desroux' no tiene correspondencia en la traducción italiana, y el verso 'que tant se desconforta' (adaptado en el texto inglés en 'and in him-self took so gret hevinesse', v. 812, y en catalán en 'que per dol e vida maltractada', v. 783) se convierte en italiano en dos versos: 'el giovane pigliò tanto disconforto,/per questa donna disperato e tristo' (vv. 880-881).

Sin duda la opción métrica de las traducciones de Chartier no obedece a razones prácticas, como se puede observar en la versión catalana. Es cierto que, dada la proximidad entre francés y catalán, a veces las dos sílabas adicionales respecto al octosílabo francés permiten el traslado palabra por palabra, pero son casos excepcionales, pues uno de los problemas más frecuentes del traductor es la obtención de sólo siete, ocho o nueve sílabas. En consecuencia, el decasílabo catalán se completa con pequeñas modificaciones o adiciones. Además, las octavas del traductor, de versos decasílabos con cesura masculina en la cuarta sílaba, son cadenoencadenadas 
$(\mathrm{ABABCDCD})$ y presentan la distinción entre rimas masculinas (agudas) y femeninas (llanas). La alternancia de rimas llanas y agudas, junto con la ampliación de tres a cuatro rimas, dificulta el traslado literal del sistema rímico del original, como se puede observar en la estrofa XCVIII citada: en el primer verso, el sintagma añadido 'ne quina via' es necesario para completar el cómputo silábico y para obtener rima llana; en los versos segundo y tercero, la adición, respectivamente, de un determinante ('aquell') y de un adverbio y un pronombre ('certes' y 'res') permiten llevar a cabo una traducción literal convirtiendo las ocho sílabas del francés en diez; en el tercer verso, además, el traductor necesita una palabra que rime con 'via' (primer verso), de ahí que traduzca 'sa dame' por 's·aymia'; el recuento silábico también determina en parte la traducción 'aux dances' por el binomio 'en dançar e pler' del cuarto verso; la rima llana y el cómputo del quinto verso explican la traducción de 'on me rapporta' por una perífrasis en pasiva ('nova·m fou reportada'); en el verso siguiente, el adjetivo 'desroux' ('rotos') se retoma en catalán con el verbo 's'ach romputs' acompañado del adverbio 'malament'; en el séptimo verso, la necesidad de rima femenina, entre otros factores, condiciona la adaptación del verso, que no sigue textualmente el francés; y, finalmente, en el último verso de la octava, el adjetivo 'courroux' es traducido por un par de sinónimos, 'tristor e turment'.

La cesura masculina del decasílabo es una constricción métrica más, que obliga a acabar en palabra aguda el primer hemistiquio del verso -las cuatro primeras sílabas-. Así, en el tercer verso de la octava XCVIII, el francés 'mais a sa dame' no puede trasladarse literalmente en un hipotético 'mas a sa dama' porque este sintagma acaba en palabra llana, y es, pues, con el adverbio 'res' que se obtiene la palabra aguda necesaria para completar la cesura.

En la traducción catalana, las modificaciones más importantes se producen 
especialmente cuando el verso requiere rima llana, y, por tanto, no se puede aprovechar la aguda del original - que en catalán normalmente sería también aguda-, o bien cuando per el cómputo silábico del verso la traducción totalmente transpositiva es imposible. En muchos casos intervienen ambos factores y, de entre la gama de recursos del traductor catalán para obtener el recuento silábico y adecuar la rima, destacan los pares de sinónimos, las perífrasis y los ripios.

Los grupos de sinónimos eran un recurso característico de las traducciones medievales del latín para traducir un único término del original, una técnica que se implantó en las traducciones entre romances (Wittlin 1991). Los binomios sinonímicos de la traducción catalana suelen conservar la palabra francesa en muchas ocasiones, como en el cuarto verso de la octava XCVIII, donde 'dances' es traducido por 'dançar e pler'. O en el ejemplo siguiente, en que el verbo francés 'rire' se convierte en 'riure y solaç':

Le rire pour le lermoyer riure y solaç per lo legramajar

No obstante, a veces el término francés original no queda recogido en la traducción, como sucede con 'courroux' (v. 784, octava XCVIII), traducido por 'tristor e turment', o con la palabra 'larmes' del verso citado a continuación, que se convierte en el binomio 'dols e plors':

Par les larmes que des yeulx yssent ab dols e plors que dels ulls fa surtir

La técnica de coordinación de sinónimos es utilizada también por los traductores 
inglés e italiano: por ejemplo, la palabra 'corroux' citada, es traducida en italiano por el binomio 'de cruccio e d'ira'; y, en la traducción inglesa, la palabra 'maistresse' de la primera octava del poema francés ('la mort me tolly ma maistresse', v. 6) es traducida en inglés 'my lady and maitresse' ('The deeth hath take my lady and maistresse', v. 34). Los binomios sinonímicos eran también un recurso retórico habitual en la lírica medieval, por eso no es difícil encontrar, en la poesía catalana de la época, ejemplos idénticos o similares a los de la traducción catalana: ${ }^{11}$

riure y solaç per lo legramajar (v. 12)

‘solaç e xant, deport e ris’ (Andreu Febrer, Rao 59.12, v. 98)

‘solas ne joy [...]' (Martí Garcia, Rao 73.3, v. 14)

$\sim$ ell era mort de tristor e turment (v. 784)

'[...] dan e turment' (Ausiàs March, Rao 94.116, v. 19)

'[...] pena, dol e turment' (Lleonard de Sos, Rao 175.15, v. 20)

$\sim \quad$ ab dols e plors que dels ulls fa surtir (v. 24)

'ab plors e dols [...]' (J.B. de Masdovelles, Rao 103.42, v. 4)

'[...] de dol e plors' (B. H. de Rocabertí, Rao 149.2, v. 190)

Las perífrasis son otro procedimiento habitual en la traducción catalana, especialmente las verbales:

De dicter et de rimoyer de ffer dictats e de cobles rimar

(v. 10)

\footnotetext{
${ }^{11}$ Todas las poesías catalanas citadas en este artículo se acompañan de una referencia entre paréntesis, que contiene el nombre del autor, el código Rao del poema y la numeración de los versos. Asimismo, en todos los casos la edición citada es la del RIALC (Repertorio informatizzato dell'antica letteratura catalana).
} 
En el primer ejemplo, el verbo francés 'dicter' es traducido por el verbo 'fer' acompañado de un complemento que recoge la raíz léxica de verbo francés ('dicter' 'dictats') y 'rimoyer' se convierte en su equivalente 'rimar' seguido de un complemento directo ('cobles'). En el segundo caso, la perífrasis 'pena sosté' traduce el sentido del verbo original 'languist', pero no mantiene la raíz léxica de la palabra francesa en ninguna de las catalanas. A su vez, los traductores inglés e italiano también recurren en muchas ocasiones a las perífrasis: la traducción inglesa presenta una solución similar a la catalana para el verso 10 francés ('de dicter et de rimoyer'), 'either to ryme or dytees for to make' (v. 38) y, en la octava XCVIII citada, el verso 'tant se desconforta' (v. 783) se convierte en italiano en la perífrasis 'pigliò tanto isconforto'. Finalmente, como en los grupos de sinónimos, las perífrasis verbales utilizadas por el traductor catalán se documentan también en la poesía catalana medieval:

$\sim$ de ffer dictats e de cobles rimar (v. 10)

‘los trobadors a fer dictats acuyten’ (Ausiàs March, Rao 94.113, v. 46)

'vers ne dictat d'amor fer no podia' (B.H. de Rocabertí, 149.2, v. 49)

$\sim$ qui pus tost mor, cert, menys pena sosté (v. 264)

'com no so plant de pena sostenguda' (Ausiàs March, Rao 94.109, v. 6)

'merexen be tal pena sostenir' (Anónimo, Rao 0.59, v. 80)

Además de sinónimos y perífrasis, son abundantes en la traducción catalana los ripios, palabras para rellenar el verso que no corresponden a ningún término del 
original. Su función principal es posibilitar la adecuación de la rima, como en el sintagma 'ne quina via' de la octava XCVIII (v. 777). La traducción inglesa también cuenta con este tipo de ripios, como se advierte en esta misma octava: el sintagma 'for anguissh and for payne' (v. 811), que además es un binomio sinonímico, se añade para completar el verso, y, en el verso final (v. 813), 'within a day or twayne' es una adaptación motivada por la necesidad de adecuar la rima con 'payne'. El contenido del texto francés no queda comprometido por la adición de ripios, pero, al menos en la traducción catalana, es en estos versos menos literales donde aparecen más similitudes con la lírica amorosa de mediados del siglo XV. El caso más evidente es el de las rimas, pues el traductor sólo aprovecha las rimas francesas en cuarenta pares de versos, lo cual significa que, de los ochocientos versos del poema de Chartier, el 90\% de las palabras rima catalanas son innovación del traductor. La semejanza entre el catalán y el francés hubiera permitido más rimas coincidentes, pero la opción métrica escogida por el traductor catalán lo impide. En efecto, el rimario de la traducción es muy parecido al de cualquier poeta catalán de mediados del siglo XV, como se puede observar en estos ejemplos:

$\sim$ Depuis je ne sceu qu'il devint $[\ldots]$ Mais a sa dame n'en souvint mas, certes, res no·n membrà a s'aymia (vv. 777 y 779) Rimas vialaymia:

'per sobresalt qui·m ve de vós, m'aymia:/del no amar desalt ne té la via' (Ausiàs March, Rao 94.82, vv. 14-15)

'Mos vulls, qui m'an donat tant mal, m'aymia//entrar m'an ffet en perillosa via' (Joan Berenguer de Masdovelles, Rao 103.136, vv. 9 y 12) 
$\sim$ Si gracïeuse maladie

$[\ldots]$

Mais il siet bien que l'on le die
Tan graciós mal ni de semblant pasta

mas ell està bé lo dir al qui u tasta

(vv. 265 y 267)

Rimas tasta y pasta y pasta/tasta:

'fruit del deleyt del qual negu no tasta' (Jordi de Sant Jordi, Rao 164.1, v. 16)

'mes dubte·m molt que prenent semblant pasta' (Mossèn Avinyó, Rao 10.5, v.

'Sol per a vós bastà la bona pasta/[...]/mas compliment dona Teresa.l tasta' (Ausiàs March, Rao 94.43, vv. 25 y 28)

En definitiva, el traductor catalán trasladó con fidelidad el discurso de Alain Chartier, pero lo pasó por el tamiz de la lengua poética a la cual estaba habituado. Tanto es así, que uno de los cinco manuscritos que ha transmitido la traducción catalana la atribuye a Ausiàs March: al responsable de esta atribución errónea debía parecerle que la obra tenía las características del gran poeta modelo del cuatrocientos. ${ }^{12}$ Asimismo, en la versión italiana se puede apreciar que el traductor estaba siempre, 'inevitabilmente, con l'occhio volto al padre Dante' (Sansone 1997: 8), y el gran éxito que tuvo la traducción inglesa se debe en parte al hecho de que fue atribuida a Geoffrey Chaucer. ${ }^{13}$ Escribir en verso en el siglo XV, aunque se tratase de una traducción, llevaba implícita la adopción de unas formas métricas limitadas e implicaba la necesaria remisión a una lengua poética, unas fórmulas, unos estilemas y un vocabulario que se imponían en el

\footnotetext{
${ }^{12}$ Se trata del manuscrito $\mathrm{n}^{\circ} 10$ de la Biblioteca de Catalunya (Barcelona), un descriptum de otro códice (París, Bibliothèque Nationale, ms. esp. 225) que no contiene este error de atribución.

${ }^{13} \mathrm{La}$ traducción inglesa se conserva en siete manuscritos y cuatro ediciones impresas; algunos de estos manuscritos contenían mayoritariamente obras de Geoffrey Chaucer y en el colofón de una de las ediciones se atribuyó la traducción a este autor, lo cual garantizó el éxito posterior del poema. Estas cuestiones se explican con detalle en: Kinch 2006. Por otro lado, la traducción inglesa incorpora un prólogo y un epílogo del traductor en royal stanza, estrofas de siete versos cuya creación se atribuye tradicionalmente a Geoffrey Chaucer.
} 
traductor, ya fuese conscientemente o de manera automática. Esta noción de la traducción poética continuaba siendo vigente a principios del siglo XVI, como demuestran algunas traducciones castellanas. Por ejemplo, la traducción parcial del soneto de Petrarca S'amor non è de Pedro de Cartagena, contenida en el Cancionero General de 1511, es en octosílabos. ${ }^{14}$ Asimismo, son en quintillas de octosílabos las dos primeras traducciones de los Triomphi de Petrarca, la íntegra de Antonio de Obregón (1512) y la del Triumfo de Amor, de Álvar Gómez (c. 1515). En 1554 se imprimió una tercera traducción de los Triomphi, la de Hernando de Hozes, que reproducía las terzine de versos endecasílabos del original. En el prólogo a su traducción, Hernando de Hozes justificó su opción métrica puntualizando que las obras de Boscán, Garcilaso y otros autores avalaban el uso del endecasílabo y la imitación del verso italiano 'al pie de la letra':

Pero en fin me pareció que era mejor auenturarme a este inconveniente [traducir en terzine], que no a contradecir la opinión de tantos, como lo que el día de oy son de voto, que al pie de la letra se imite también en esto la manera del verso italiano, como en todas las otras cosas, puesto caso que no es justo que ninguno condene por malo aquello que don Diego de Mendoça, y el secretario Gonzalo Pérez, y don Ioan de Coloma y Garci Lasso de la Vega, y Ioan Boscán y otras personas doctas tienen aprobado por bueno'.

(Hernando de Hozes, fol. vi, apud Recio 2007: 169)

No obstante, a principios de siglo las tendencias eran otras, y el octosílabo de las primeras traducciones castellanas de los Triomphi respondía a la moda de la lírica cancioneril, en la cual se sumergió especialmente Álvar Gómez: su traducción, que

\footnotetext{
${ }^{14}$ Para esta y otras traducciones castellanas del soneto S'amor non è, véase Marías en prensa.
} 
diluye a Petrarca 'a la maniera cancioneril', es representativa de como una 'pieza elaborada en un sistema entra en un código poético distinto' (Rico 1978: 330 y 331). Algo parecido sucede con las dos traducciones castellanas de Ausiàs March, la de Baltasar de Romaní (1539) y la de Jorge de Montemayor (1560), que «reflejan perfectamente el estado de la poesía española antes y después de Garcilaso» (Micó 2002: 90): Romaní, quizás porque era valenciano, intenta reproducir el decasílabo con cesura masculina en la cuarta sílaba de March, mientras que Montemayor utiliza el endecasílabo italiano introducido por Boscán y Garcilaso. ${ }^{15}$ Este 'antes de' se observa también en la primera traducción castellana en verso del Inferno de Dante, hecha por Fernández de Villegas, y publicada en 1515. Villegas adapta la Commedia de Dante en coblas de arte mayor, ocho dodecasílabos que riman ABBA-ACCA, con dos hemistiquios de seis sílabas. El traductor confiesa en el prólogo haber probado de traducir en terza rima y argumenta por qué desistió en el intento:

Yo prové a los fazer [los versos] ansí en tercetos, la cual manera no es en nuestro uso y parecíame una cosa tan desdonada que lo dexé.

(Fernández de Villegas 1515: fol. aiij) ${ }^{16}$

A pesar de las dificultades que acarreaban las coplas de arte mayor, detalladas en el prólogo, Villegas justificaba así su elección:

[La versificación de Dante es] conforme al trobar castellano de arte mayor en que Juan de Mena escrivió el su Laberinto de las Trezientas coplas, y porque aquella manera es tan conforme al verso suyo y tanbién porque es más grave y de mayor resonancia como

\footnotetext{
${ }^{15}$ Para las traducciones castellanas de Ausiàs March, véase Riquer 1946 y Cabré 2002.

${ }^{16}$ En las citaciones de esta obra, puntúo y acentúo el texto.
} 
convenía a tan grave auctor, yo fize esta traslación en aquella forma de trobar que propiamente es verso heroyco $[\ldots]$

(Fernández de Villegas 1515: fol. aiij)

En efecto, la copla de arte mayor era la forma métrica, adecuada para los asuntos 'graves', con la cual la tradición lírica castellana se había aproximado a Dante, pues habían cultivado las coplas de arte mayor Francisco Imperial (1350?-1409?), el Marqués de Santillana (1398-1458) y Juan de Mena (1411-1456), precedente el primero y exponentes los dos últimos de la denominada escuela alegórico-dantesca del siglo XV. En la elección métrica de Villegas, como él mismo menciona, fue determinante el Laberinto de Fortuna o Las trescientas de Juan de Mena, una obra con notoria influencia dantesca que en el siglo XVI fue comentada y glosada como se hacía con la Commedia de Dante. ${ }^{17}$

El tratamiento de la métrica en las traducciones medievales de La Belle Dame sans merci y en las castellanas citadas de Dante y Petrarca revela claramente la condición de producto histórico inherente a toda traducción. En el siglo XV, también a principios del XVI, la pauta que regía la traducción poética era conseguir un texto de calidad, formalmente perfecto, fiel a las normas -métricas y estilísticas- y a las modas imperantes en la tradición poética del traductor. ${ }^{18}$ El grado de fidelidad al sentido y de literalidad respecto al texto de partida quedaban supeditados a esta pauta estética. Era la solución de los traductores ante la pérdida de la 'dolcezza ed armonia' del original: restituirla con la musicalidad de su propio código poético.

\footnotetext{
${ }^{17}$ El texto de Mena con glosas de Hernán Núñez fue impreso por primera vez en 1499 y se publicó una segunda edición corregida en 1505 - de la cual derivan todas las ediciones posteriores-; en 1582 aparecía la versión del Laberinto de Fortuna anotada por el Brocense. Para estas cuestiones, véase el prólogo de Carla de Nigris a Juan de Mena 1994: LXXXV-LXXXVI.

${ }^{18}$ Véanse conclusiones similares en Sansone 1997: 9 a propósito de la traducción italiana de La Belle Dame sans merci y en Recio 1998: 19-20 sobre el Triumfo de Amor de Álvar Gómez.
} 


\section{Obras citadas}

Alighieri, Dante, 1997. Il Convivio (Roma: Salerno Editrice).

Alighieri, Dante, 1991. De vulgari eloquentia (Milán: Garzanti Editore).

Badia, Lola, 1994. 'La legitimació del discurs literari en vulgar segons Ferran Valentí', en Intel-lectuals i escriptors a la Baixa Edat Mitjana, ed. Lola Badia y Albert Soler (Barcelona: Curial - Publicacions de l'Abadia de Montserrat), pp. 161-184.

Cabré, Lluís, 2002. 'Algunes imitacions i traduccions d'Ausiàs March al segle XVI', Quaderns. Revista de Traducció, 7, pp. 35-57.

Cabré, Lluís, 2007. 'Andreu Febrer, fabbro i lector', en From the Cancioneiro da Vaticana to the Cancionero general: Studies in Honour of Jane Whetnall, ed. Alan Deyermond y Barry Taylor (Londres: Queen Mary, University of London), pp. 103-114.

Calin, William, 1994. The French Tradition and the Literature of Medieval England (Toronto: University of Toronto Press).

Fernández de Villegas, Pero, 1515. La tradución del Dante de lengua toscana en verso castellano (Burgos: Fadrique Alemán de Basilea) (ejemplar consultado: Biblioteca de la Universitat de Barcelona, 07 CM-621). 
Gallina, Annamaria, 1974-1988. Dante Alighieri, Divina Comèdia. Versió catalana d'Andreu Febrer (Barcelona: Barcino).

Gascón, Elena, 1992. 'Enrique de Villena: ¿castellano o catalán?’, en Actas del X Congreso de la Asociación Internacional de Hispanistas. Barcelona 21-26 de agosto de 1989, ed. Antonio Vilnova (Barcelona: PPU), vol. I, pp. 195-206.

Kinch, Ashby, 2006. "«To corecte in any part or alle»: Some Problems in the Transmission of the Middle English La belle dame sans mercy', Mediaeval Studies, 68: 333-350.

Laidlaw, J.C, 1974. The Poetical Works of Alain Chartier (Cambridge: Cambridge University Press).

López de Mendoza, Iñigo (Marqués de Santillana), 1997. Comedieta de Ponza, sonetos, serranillas y otras obras (Barcelona: Crítica).

Marfany, Marta, 2010. 'La traducció catalana medieval de La Belle Dame sans merci d'Alain Chartier', en Translatar i transferir. La transmissió dels textos i el saber (12001500), ed. Anna Alberni, Lola Badia y Lluís Cabré (Santa Coloma de Queralt: Obrador Edèndum), pp. 179-188.

Marías, Clara, en prensa. 'Translation and Imitation in Early Modern Spanish Poetry: A Case Study of Petrarch's Sonnet S'amor non è', en las actas del XIV Forum for Iberian Studies. The Limits of Literary Translation. 24-25 June 2010. 
Mena, Juan de, 1994. Laberinto de Fortuna y otros poemas (Barcelona: Crítica).

Micó, José María, 2002. 'Verso y traducción en el Siglo de Oro', Quaderns, Revista de Traducció, 7, pp. 83-93.

Parera, Raquel, 2010. 'La versió d'Andreu Febrer de la Commedia de Dante: recursos del traductor', en Translatar i transferir. La transmissió dels textos i el saber (12001500), ed. Anna Alberni, Lola Badia y Lluís Cabré (Santa Coloma de Queralt: Obrador Edèndum), pp. 161-178.

Pascual, José A, 1974. La traducción de la Divina Commedia atribuida a D. Enrique de Aragón. Estudio y edición del Infierno (Salamanca: Universidad de Salamanca).

Piaget, Arthur, 1941. Oton de Grandson. Sa vie et ses poésies (Lausanne: Payot).

Recio, Roxana, 1998. El Triumfo de Amor de Petrarca traducido por Álvar Gómez (Barcelona: PPU).

Recio, Roxana, 2007. 'Metodología y enseñanza de la traducción en el siglo XVI: los textos de Castillo y Álvar Gómez’, Journal of Iberian Studies, 9: 161-174.

RIALC, Repertorio informatizzato dell'antica letteratura catalana, ed. Università di Napoli Federico II.

http://www.rialc.unina.it. Consultado el 15 de junio de 2010. 
Rico, Francisco, 1978. 'De Garcilaso y otros petrarquismos', Revue de Littérature comparée, 52: 325-338.

Riquer, Martí de, 1946. Traducciones castellanas de Ausiàs March en la Edad de Oro (Barcelona: Instituto Español de Estudios Mediterráneos).

Riquer, Martí de, 1983. Alain Chartier. La belle dame sans merci. Amb la traducció catalana del segle XV de fra Francesc Oliver (Barcelona: Quaderns Crema).

Sansone, Giuseppe E., 1997. La Dama sanza merzede (Roma: Zauli Editore).

Symons, Dana M, 2004. Chaucerian Dream Visions and Complaints (Michigan: Medieval Institute Publications).

Wittlin, Curt J., 1991. Repertori d'expressions multinominals $i$ de grups de sinònims en traduccions catalanes antigues (Barcelona: Institut d'Estudis Catalans). 